\title{
Thermal annealing of gold coated fiber optic surfaces for improved plasmonic biosensing
}

\author{
Iulia Antohe $(\text { Arghir })^{1}$, Koen Schouteden ${ }^{2}$, Peter Goos ${ }^{1,3}$, Filip Delport ${ }^{1}$, Dragana Spasic ${ }^{1}$ \\ and Jeroen Lammertyn ${ }^{1 *}$ \\ ${ }^{1}$ KU Leuven, Department of Biosystems, MeBioS, Willem de Croylaan 42, B-3001 Leuven, \\ Belgium \\ ${ }^{2}$ KU Leuven, Department of Physics and Astronomy, Solid-State Physics and Magnetism \\ Section, Celestijnenlaan 200D, B-3001 Leuven, Belgium \\ ${ }^{3}$ University of Antwerp, Department of Engineering Management, Prinsstraat 13, B-2000 \\ Antwerpen, Belgium
}

*Author to whom correspondence should be addressed:

E-mail: jeroen.lammertyn@biw.kuleuven.be

Phone: +32 (0) 1632 1459; Fax: +32 (0) 16322955

\begin{abstract}
The morphological properties of thin gold (Au) films sputtered onto fiber optic (FO) substrates play an essential role in the overall performance of the sensing devices relying on surface plasmon resonance (SPR) effects. In this work, the influence of thermal treatments on the structural changes of the Au layer coated on the FO-SPR sensors, and consequently on their plasmonic biosensing performance, was systematically investigated. First, the sensors were exposed to different annealing temperatures for different durations and their sensitivity was evaluated by refractometric measurements in sucrose dilutions. The attained results suggested the optimal annealing conditions that were further validated using a split-plot experimental design statistical model. Room-temperature scanning tunneling microscopy (STM) imaging of the FO substrates revealed changes in the granular surface texture of the thermally treated $\mathrm{Au}$ films that could be linked to the observed increase in sensitivity of the treated sensors. The FO sensors, annealed under optimal conditions, were finally tested as label-free aptamer - based biosensors for the detection of Ara $h 1$ peanut allergen. Remarkably, the results demonstrated a superior biosensing performance of the thermally treated FO sensors, as the limit of detection (LOD) was improved with up to two orders of magnitude compared to a similar non-annealed FO-SPR sensor. This significant increase in sensitivity represents a major step forward in the facile and cost-effective preparation of FOSPR sensors capable of label-free detection of various biomolecular targets.
\end{abstract}

Keywords: Fiber optic - surface plasmon resonance (FO-SPR); gold ( $\mathrm{Au}$ ) thermal annealing; refractometric measurements; split-plot experimental design; scanning tunneling microscopy (STM); Ara h 1 peanut allergen label-free detection. 


\section{Introduction}

Surface plasmon resonance (SPR) biosensors are powerful optical tools in biotechnology and medicine because they can be used for sensitive detection and real-time monitoring of (bio)chemical interactions [1]. Furthermore, coupling SPR to fiber optic (FO) technology triggered notable advancements in the field, by offering attractive advantages over the classical prism-based SPR platforms, such as simplicity, portability, cost-effectiveness and miniaturization [2]. FO-SPR sensors were successfully used already in a variety of applications, such as screening for bacterial infections [3], monitoring of environmental water pollution [4] or checking for specific toxins [5] and allergens [6] in food, among others. However, their performance still needs to be improved in order to match traditional prismbased SPR sensors, and consequently, to switch from laboratory proofs-of-principle to commercial products.

Different strategies were employed so far in an attempt to enhance the overall sensing performance of the FO-SPR system [7], [8], [9]. Many of these methods rely on improving the quality and/or optimizing the morphology of the thin gold ( $\mathrm{Au}$ ) film sputtered on the FO silica core within the sensitive zone, with a reported thickness around $50 \mathrm{~nm}$ for an optimal SPR excitation [10]. One way is to simply use an organosilane compound, such as (3aminopropyl)trimethoxysilane (APTMS) [11] or (3-mercaptopropyl)trimethoxysilane (MPTMS) [12], as an efficient adhesion promoter for the Au layer attachment to the FO. Such protocols allow the design of FO-SPR sensors with considerably improved chemical stability and mechanical robustness [12], while mainly not affecting other performance quantifiers of the device, such as sensitivity or limit of detection (LOD). These two parameters can be further improved by designing "sandwich"-like bioassays, in which secondary recognition elements are added, either free or labelled with $\mathrm{Au}$, silica and/or magnetic nanoparticles (NPs) [6], [13]. Although such methodologies are frequently used, they complicate the overall handling procedures by increasing the analysis time and assay implementation costs.

An alternative approach involves immobilization of chemically synthesized nanomaterials directly on the FO sensitive zone, thereby altering the Au surface morphology [7], [14], [15]. Such approaches of deliberately diminishing the smoothness of the as-deposited plasmonic film, might be promising in the development of FO-SPR instruments because, as already well-established with the planar SPR substrates [16], they could enhance the sensor's refractometric response, or eventually they could enable additional functionalities, like multiplexing [17] or localized SPR (LSPR) [18], [19], thus allowing parallel analysis of different target analytes [20] or detection of tiny biomolecules in low amounts [16], respectively. The optimized wet chemistry protocols enable the synthesis of a wide variety of nanomaterials (i.e. spheres, rods, triangles) possessing great plasmonic properties. In most of the cases, FO surfaces functionalized with amino- or thiol- groups attract the nanomaterials via either covalent or electrostatic linkage strategies [11], [21]. However, these protocols are not always easy to implement due to the potential limitations in polydispersity and reproducibility, mainly associated with the nanomaterials aggregation during the process [22]. To overcome these drawbacks, advanced nanopatterning techniques, such as optical or electronic lithography were introduced, allowing the texturing of FO surface with highlyordered arrays of nanostructures exhibiting well-defined shapes and sizes [23], [24], [25]. Although these methods provide an excellent control over the nanostructures aspect and their arrangement on the substrate, they remain complex, expensive and time-consuming approaches, as well as still very challenging to be applied on the non-planar FO substrates.

In contrast, a cost-effective alternative of texturing the curved FO surface is to simply coat it in optimized conditions with the thin $\mathrm{Au}$ plasmonic film, and to optionally expose it to specific surface treatments in order to induce further morphological changes [26]. Thermal 
evaporation of metals to form nanostructured films on SPR planar substrates is currently one of the simplest and cheapest pathways to produce plasmonic sensors with LSPR ability [27], [28]. Furthermore, additional studies showed that specific thermal exposures of these thin metallic layers (e.g. Au) allow the formation of metallic islands subsequently causing strong plasmonic behaviors due to the presence of "hot spots" that form along the small gaps in the metallic film [29], [30], [31]. The first experiments of modifying a FO tip in this way were reported by Mullen et al., although the obtained device was only used for surface-enhanced Raman scattering (SERS) measurements [32]. Moreover, the influence of such simple thermal treatments on the morphology of the Au layer sputtered on the FO substrate, as well as on its plasmonic (bio)sensing performance, has been poorly studied experimentally, being mainly subject to theoretical modelling [33].

In this work, the influence of thermal annealing on the sensing behavior of FO-SPR sensors is experimentally investigated by refractometric measurements in sucrose dilutions. The optimal annealing conditions are further validated using a split-plot experimental design statistical model. Furthermore, the morphology of the Au film before and after the annealing steps is characterized by room-temperature scanning tunneling microscopy (STM) of the coated FO sensors. To the best of our knowledge, this is the first attempt of employing such a probing tool in analyzing curved surfaces of thin fibers, although yet limitedly, the use of a conventional atomic force microscope (AFM) for similar purposes was already reported [34]. The optimally annealed FO-SPR sensor is finally tested for the label-free detection of Ara $\mathrm{h} 1$ peanut allergen.

\section{Materials and Methods}

2.1 Chemicals. All chemical products used in this work were of high-quality analytical grade $(99.99 \%$ purity, unless otherwise specified). The dilutions were entirely prepared with ultra-clean deionized water (DIW) purified by a Milli-Q 50 system (Millipore, USA). A molecular nitrogen $\left(\mathrm{N}_{2}\right)$ gas stream with an estimated volumetric flow rate of $35 \mathrm{~m}^{3} / \mathrm{h}$ was used to dry the samples during the different processing steps.

Acetone, sulfuric acid $\left(97 \% \mathrm{H}_{2} \mathrm{SO}_{4}\right)$ and acetic acid were purchased from Chem-Lab, Belgium. Hydrogen peroxide $\left(30 \% \mathrm{H}_{2} \mathrm{O}_{2}\right)$, anhydrous toluene, methanol, MPTMS, trizma base, sodium dodecyl sulfate (SDS) and ethylenediaminetetraacetic acid (EDTA) were supplied by Sigma-Aldrich, Belgium. (1-mercapto-11undecyl)tri(ethylene glycol) $\left[\mathrm{SHC}_{11}(\mathrm{PEG})_{7}\right]$ was obtained from Polypure, Norway. $\mathrm{D}(+)$-sucrose and glycine were acquired from Fisher Scientific, Belgium. Dithiothreitol (DTT), potassium phosphate monobasic $\left(\mathrm{KH}_{2} \mathrm{PO}_{4}\right)$ and dibasic $\left(\mathrm{K}_{2} \mathrm{HPO}_{4}\right)$ were purchased from Applichem, Germany. Purified natural Ara h 1 was supplied by Indoor Biotechnologies Limited, United Kingdom. The thiolated anti-Ara $\mathrm{h} 1$ DNA aptamer (5'-TC GCA CAT TCC GCT TCT ACC GGG GGG GTC GAG CTG AGT GGA TGC GAA TCT GTG GGT GGG CCG TAA GTC CGT GTG TGC GAA-3') was purchased from Integrated DNA Technologies, Belgium and was shipped in its oxidized (disulfide) form. A NAP-5 column from GE Healthcare, United Stated was used for the DNA purification.

The TGK buffer ( $\mathrm{pH} 8.3$ ) was obtained by preparing an aqueous solution containing 25 $\mathrm{mM}$ trizma base, $192 \mathrm{mM}$ glycine and $5 \mathrm{mM} \mathrm{K}_{2} \mathrm{HPO}_{4}$. The TE buffer ( $\mathrm{pH} 8$ ) was prepared by mixing $10 \mathrm{mM}$ trizma base and $1 \mathrm{mM}$ EDTA in $1 \mathrm{~L}$ of DIW.

2.2. FO-SPR sensors fabrication. The FO-SPR sensors were prepared using a previously described protocol [12]. In brief, a TEQS multimode FO (Thorlabs, Germany) with a diameter of $400 \mu \mathrm{m}$ was split into fragments with a consistent length of $3.6 \mathrm{~cm}$. Then, a 
sensitive SPR zone of $0.6 \mathrm{~cm}$ was constructed at one side by mechanically removing the FO jacket and dissolving the polymer cladding in acetone to expose the FO silica core. The core was then carefully dried further with dust-free tissues. The hydroxyl groups $\left(\mathrm{OH}^{-}\right)$on the FO core surface were next activated by immersing the samples in a piranha solution $(3: 7 \% \mathrm{v}: \mathrm{v}$ $\mathrm{H}_{2} \mathrm{O}_{2}: \mathrm{H}_{2} \mathrm{SO}_{4}$ ) for 15 min. The FO-SPR sensors were thoroughly rinsed with DIW and methanol and afterwards dried under $\mathrm{N}_{2}$ gas flow.

In a second stage, the prepared sensors were subjected to a silanization process for improving the $\mathrm{Au}$ adhesion on the FO silica core, using an optimized wet chemistry approach as previously reported [12]. In this context, the samples were ultrasonicated for $2 \mathrm{~h}$ in anhydrous toluene, containing $0.3 \mathrm{M}$ acetic acid and $8 \mathrm{mM}$ MPTMS, and then the silanized sensors were cleaned in methanol and dried using $\mathrm{N}_{2}$ gas stream. In the end, the silanized sensors were coated by a thin Au layer $(\sim 50 \mathrm{~nm})$ using a sputter coater (Quorum Q150T ES, UK). The DC plasma was engaged for $7 \mathrm{~min}$ at $21 \mathrm{~mA}$ in Ar atmosphere kept at $2.5 \cdot 10^{-2}$ mbar and the FO sensors were installed on a rotating stage $(100 \mathrm{rpm})$ to improve the $\mathrm{Au}$ coverage during the sputtering process, while the deposited thickness was real-time monitored using the built-in quartz crystal resonator. For comparison purpose, reference (non-silanized) FO sensors were directly coated by $\mathrm{Au}$ in similar conditions without any prior silanization step.

Finally, both reference and silanized FO sensors were put in an oven (Memmert $\mathrm{GmbH}$, Germany) and exposed to an annealing treatment at 130,180 and $230^{\circ} \mathrm{C}$, for 2,7 and $12 \mathrm{~h}$, after which they were slowly cooled down to room temperature.

2.3. FO-SPR refractometric measurements. The FO-SPR sensitivity was tested by performing refractive index (RI) measurements in serial sucrose dilutions $(0,2,4,8,12 \%$ $\mathrm{w} / \mathrm{w}$ ). The Brix values (see Table S1 from the supporting information) of the prepared sucrose dilutions were checked with a digital refractometer (Atago Palette PR-32, Japan). The interchangeable FO-SPR sensors were typically inserted into adequate SMA connectors (Avantes, USA) and mounted on a computer-controlled SPR measuring platform [12]. The FO sensors were kept one minute in each sucrose solution during the RI measurements, unless otherwise specified. The FO-SPR sensitivity was afterwards evaluated by plotting the SPR wavelength shifts as a function of the RI values of each sucrose solution, followed by linearly fitting the obtained calibration curve (see Figure S1 from the supporting information). Three FO-SPR sensors were prepared per specified condition (annealing temperature and duration) and the sensitivity values were calculated before and after each thermal treatment for both reference and silanized FO sensors (see Table S2 from the supporting information).

2.4. Split-plot statistical analysis procedures. A triplicated split-plot $3 \times 3 \times 2$ factorial experimental design model, constructed using the JMP statistical package (SAS Institute Inc., Cary, NC) [35], [36], was used (i) to study and model the impact of the factors sensor type (reference versus silanized), annealing temperature and annealing duration on the increase in sensitivity, and (ii) to identify settings for these three factors that result in large FO-SPR performance improvements. The experiment involved thus one 2-level categorical factor and two 3-level quantitative factors, the levels of which are summarized in Table 1.

The experimental design, involving 54 tests in total, was of the split-plot type because, thrice for each annealing temperature studied, three reference FO-SPR sensors and three silanized FO-SPR sensors were placed in an oven simultaneously, leading to nine groups of six dependent tests. One reference sensor and one silanized sensor were removed after $2 \mathrm{~h}$, another reference sensor and another silanized sensor were removed after $7 \mathrm{~h}$ and the final two sensors were removed after $12 \mathrm{~h}$. Consequently, the annealing temperature acted as a 
whole-plot factor, while the annealing time and the sensor type were sub-plot factors. Eventually, the split-plot response surface analysis indicated that the dependence between tests based on the sensors placed in the same oven was negligible.

Three FO-SPR sensors of each type were prepared per annealing condition (see section 2.3) and their corresponding sensitivity values were further used to perform the split-plot experimental design simulation. In this experiment, the intra-whole-plot correlation and the intra-block correlation were estimated to be zero. The final and simplified experimental design model was therefore obtained by taking into consideration the full second-order response surface model of the two quantitative factors and the categorical factor, while dropping out the other insignificant terms.

Table 1. Factors and factor levels used in the triplicated split-plot experimental design.

\begin{tabular}{l|l|l} 
Factor type & \multicolumn{1}{|c}{ Factor } & \multicolumn{1}{|c}{ Level } \\
\hline \multirow{2}{*}{ Categorical } & \multirow{2}{*}{ FO-SPR sensor type } & Reference \\
\cline { 3 - 3 } & & Silanized \\
\hline \multirow{4}{*}{ Quantitative } & \multirow{2}{*}{ Temperature $\left({ }^{\circ} \mathrm{C}\right)$} & 130 \\
\cline { 3 - 3 } & & 180 \\
\cline { 3 - 3 } & \multirow{2}{*}{ Time (h) } & 230 \\
\cline { 3 - 3 } & & 12
\end{tabular}

2.5. FO-SPR surface observations. A room-temperature STM setup (NanoScope II, Digital Instruments) operating in air was used to observe the topographical changes in the $\mathrm{Au}$ film structure before and after exposing the FO-SPR sensors to the different temperature treatments. In this context, the FO substrate was mounted horizontally on the STM holder.

The STM measurements were performed in constant-current mode using a mechanically cut Pt/Ir tip (10\% Ir, MaTeck GmbH, Germany) mounted on a piezoelectric scanner. The tipsample approach was done in two steps. First, the STM tip was positioned vertically above the horizontally mounted FO substrate and - using an optical microscope - the STM tip was manually approached towards the mirror-like FO substrate until the STM tip was observed to be in close proximity with its reflection on the FO substrate surface. To avoid as much as possible tip convolution effects, which occur more pronounced with increasing tilt/curvature of the substrate surface, care was taken to have the STM tip positioned on the top of the cylindrical FO. Second, using a feedback system, the STM tip was brought very close (around $1 \mathrm{~nm}$ ) to the conducting Au surface in order to detect the tip - surface tunneling current that is highly sensitive to the surface topography variations [37], [38]. Next, images were acquired by measuring the vertical extension/contraction of the piezo tube (in order to keep the tunneling current constant) as the tip was scanned horizontally by the piezo tube across the curved FO substrate, yielding a three-dimensional (3D) image of the sample topography. Typical tunneling voltage and tunneling current setpoints were $0.5 \mathrm{~V}$ and $1 \mathrm{nA}$, respectively. The obtained micrographs were then subject to a planarization algorithm (lineby-line flattening operation) to account for the substrate curvature, using WSxM software (version 5.0, Nanotec, Spain) [39].

The STM images were afterwards studied using a particle analysis procedure implemented in ImageJ software (version 1.49, NIH, United States). In this context, the examined images were subject to particle identification through local maxima determination, and then exposed to a segmentation process based on a "watershed" algorithm [40]. The latter procedure allowed thus for an estimation of the mean lateral size of the Au grains, before and after the annealing treatments. 
2.6. Label-free bioassay implementation. The bioassay was implemented as previously described [6]. Briefly, the thiol modified aptamer against Ara h 1 protein was first reduced with DTT $(0.1 \mathrm{M})$ in a $0.18 \mathrm{M}$ phosphate buffer $(\mathrm{pH})$. The activated DNA sequence was further purified using a NAP-5 column to remove the salt and the unincorporated nucleotides. The pure labeled DNA was then eluted with TE buffer ( $\mathrm{pH} 8$ ).

Subsequently, the FO sensors annealed under previously reported conditions (i.e. at $180^{\circ} \mathrm{C}$ for $7 \mathrm{~h}$ ) were incubated overnight at $4^{\circ} \mathrm{C}$ in a $150 \mu \mathrm{L}$ solution containing the DNA aptamer in $0.01 \mathrm{M}$ phosphate buffer with $0.01 \% \mathrm{SDS}$ and $1 \mathrm{M} \mathrm{NaCl}$. The salt was added to maximize the DNA loading on the Au surface. After the overnight incubation, the FO sensors were washed in $0.01 \mathrm{M}$ phosphate buffer with $0.01 \%$ SDS and further backfilled using a thioalkane with seven PEG groups and a hydroxyl head group. The aptamer binding affinity and specificity tests were also addressed elsewhere [6].

Finally, the as-prepared FO-SPR aptasensors were further used for label-free detection of different concentrations of Ara $\mathrm{h} 1$ peanut allergen $(0,25,50,100$ and $200 \mathrm{nM})$ in TGK buffer. Each Ara h 1 concentration was measured three times independently, using freshly prepared FO-SPR sensors.

\section{Results and Discussion}

\subsection{Thermal annealing effects on the FO-SPR sensitivity}

In order to evaluate the effect of thermal annealing on the sensor's sensitivity, the reference and silanized FO sensors were first sputtered with $\sim 50 \mathrm{~nm}$ of $\mathrm{Au}$, followed by thermal treatments at 130,180 and $230^{\circ} \mathrm{C}$ for 2,7 and $12 \mathrm{~h}$ (see section 2.3). Subsequently, the FO-SPR sensors were used for RI measurements in serial sucrose dilutions $(0,2,4,8$, $12 \% \mathrm{w} / \mathrm{w}$ ) and generation of calibration curves (see Figure S1 from the supporting information). The obtained calibration curves were further used for extracting the sensitivity values before and after each thermal treatment and the difference between them $(\Delta)$ was depicted with bar charts in Figure 1 both for the reference (black) and silanized (red) FO sensors (see also Table S2 from the supporting information). As can be noticed, both types of sensors (i.e. reference and silanized) generated a similar output for most of the applied annealing conditions, which was also previously reported in our work [12]. Remarkably, all investigated sensors (both reference and silanized) showed an increased sensitivity after the thermal annealing procedure. This overall increase in sensitivity was most likely due to changes in the Au film morphology, occurring during the applied thermal stress and triggering the formation of semi-continuous $\mathrm{Au}$ layers. The range of investigated temperatures was already reported to induce structural changes in the thin Au films deposited onto planar substrates, allowing consequently the design of traditional SPR substrates with strong plasmonic activity [26], [41], [42]. Moreover, an increase in performance due to $\mathrm{Au}$ morphological changes was also demonstrated for curved FO substrates exposed to harsh chemical medium [12]. Here, although a larger increase in sensitivity was typically observed for a medium thermal treatment duration $(7 \mathrm{~h})$, the major sensitivity shift of more than 1000 $\mathrm{nm} / \mathrm{RIU}$ was obtained for a temperature of $180^{\circ} \mathrm{C}$ (as also strengthened by the split-plot experiment, see section 3.2). In contrast, a duration of $12 \mathrm{~h}$ generally lowered the sensitivity for all of the tested temperatures, suggesting that an excess exposure might affect the substrate's plasmonic properties.

As an intermediate conclusion, an initial graphical data analysis demonstrated substantial changes in the sensor's sensitivity for several thermal treatments, especially at $180^{\circ} \mathrm{C}$ for $7 \mathrm{~h}$, 
with a similar behavior observed between the reference and silanized FO sensors. A more sophisticated statistical analysis is presented in section 3.2.

\subsection{Split-plot experimental design analysis}

The triplicated split-plot $3 \times 3 \times 2$ factorial experimental design was implemented (see section 2.4) in order to study the thermal annealing conditions described in section 3.1. First, a matched pair t-test was carried out to evaluate the difference in sensitivity between the reference and silanized FO sensors exposed to the different thermal treatments (see Figure S2 from the supporting information). Although the $\Delta$ sensitivity of the silanized FO sensors was, on average, $46 \mathrm{~nm} / \mathrm{RIU}$ larger than that of the reference FO sensors, the two-sided t-test's pvalue of $0.40(>0.05)$ did not allow us to conclude that there was a statistically significant difference between the two types of sensors for the tested combinations of temperature and duration. The lack of significant difference between the sensor types was confirmed by a subsequent response surface modeling exercise, using the $\log \Delta$ sensitivity as a response, and the temperature, duration and sensor type as explanatory variables. In the final response surface model obtained, the treatment duration had a strongly significant quadratic effect. There was also a strongly significant interaction effect between the duration and the temperature: temperature had a positive effect on $\Delta$ sensitivity if the duration was as short as $2 \mathrm{~h}$ and a negative effect if the duration was as long as $12 \mathrm{~h}$. For durations of approximately 7 $\mathrm{h}$, the temperature had virtually no effect on $\Delta$ sensitivity. This is clearly shown in the contour plot in Figure 2A and the prediction profiler in Figure 2B. Both Figures 2A and B confirmed that a duration of about $7 \mathrm{~h}$ is optimal, and showed that the entire range of studied temperatures yielded good results for that duration. More specifically, for FO sensors that were exposed to an optimal annealing time of $7 \mathrm{~h}$, in the response surface model a $\Delta$ sensitivity between 775 and $1000 \mathrm{~nm} / \mathrm{RIU}$ was foreseen when annealing the sensors at 130 and $180^{\circ} \mathrm{C}$, while the model predicted a $\Delta$ sensitivity value exceeding $550 \mathrm{~nm} / \mathrm{RIU}$ at $230^{\circ} \mathrm{C}$. The prediction profiler shown in Figure $2 \mathrm{~B}$ predicted that at $180^{\circ} \mathrm{C}$, a maximal $\Delta$ sensitivity value of $\sim 820 \mathrm{~nm} / \mathrm{RIU}$ can be reached provided a duration of $6.7 \mathrm{~h}$ was used.

Based on the predictions obtained from the response surface model and the observed $\Delta$ sensitivity values in Figure 1, a temperature of $180^{\circ} \mathrm{C}$ and a duration of $7 \mathrm{~h}$ were selected for further application to the FO-SPR sensors. These settings guarantee a substantial increase in performance while employing a safely temperature value at the expense of a limited manufacturing time of the sensors. Due to the insignificant differences in $\Delta$ sensitivity between the reference and silanized FO sensors, there was no statistical reason to prefer one over the other. However, silanized FO sensors were eventually used in subsequent experimentation because it has been shown that the MPTMS presence improves the $\mathrm{Au}$ adhesion to the FO silica core, allowing thus the fabrication of reusable and mechanically stable FO-SPR sensors [12].

\subsection{Morphological characterization of the FO-SPR surface}

The influence of the thermal annealing on the surface morphology of the thin Au film sputtered on the FO silica core was further investigated with a room-temperature STM operating in air (see section 2.5). Based on the experimental and statistical data, as shown in the previous sections, a silanized FO sensor without (A) and with (B) a thermal treatment at $180^{\circ} \mathrm{C}$ for $7 \mathrm{~h}$ was examined using the STM (Figure 3). For the two depicted STM micrographs, the root mean square roughness $R_{R M S}$ (determined using WSxM 5.0) was 2.2 
$\mathrm{nm}$ and $2.4 \mathrm{~nm}$ for the non-annealed and annealed samples, respectively. Although no spectacular change in the surface roughness was obtained, a clear difference between the two FO substrates was observed by a direct visual inspection of the STM topographical images. Prior to the annealing process, the Au coated FO surface clearly exhibited a pronounced granular morphology (Figure 3A). The thermal exposure step gave rise to the coalescence of the Au grains into larger "island"-type structures, resulting in an increase of the average grain size lateral dimension (Figure 3B). The grains coalescence effect can be analyzed in more detail in Figures 3C and D (obtained using Image J 1.49), from which the mean value for the grain lateral size was estimated to be $\sim 59 \mathrm{~nm}$ and $\sim 82 \mathrm{~nm}$, before and after the annealing process, respectively. Interestingly, this phenomenon resembles the formation of $\mathrm{Au}$ "island"like semi-continuous domains previously reported to cause strong plasmonic behaviors [30], [31], thus possibly determining the observed sensitivity shifts of the thermally treated FOSPR sensors. STM micrographs taken after 2 and $12 \mathrm{~h}$ of annealing at $180^{\circ} \mathrm{C}$ are also complementarily shown in Figure S3 from the supporting information.

Concluding, the STM analysis revealed a strong correlation between the morphology evolution of the thin Au film with the annealing parameters, and the subsequent FO-SPR sensor's performance.

\subsection{Ara $h 1$ peanut allergen detection}

An aptamer based - bioassay against Ara $\mathrm{h} 1$ protein was implemented to evaluate the improved performances of the thermally treated FO-SPR sensor. Three non-annealed and three annealed silanized FO sensors were functionalized overnight with a thiolated anti-Ara $h$ 1 aptamer and used to detect in a label-free manner the Ara $\mathrm{h} 1$ protein in a TGK buffer (see section 2.6). The FO sensors were placed in the TGK buffer for $3 \mathrm{~min}$ before and after immersion in the target solution for $20 \mathrm{~min}$. Figure 4A shows the corresponding sensorgram obtained for an Ara h 1 concentration of $200 \mathrm{nM}$. The SPR wavelength shift generated by the thermally treated sensor $(12.58 \pm 0.69 \mathrm{~nm})$ was almost 12 times larger compared with the signal produced by the non-annealed FO $(1.09 \pm 0.35 \mathrm{~nm})$. This increase in sensitivity can be attributed to the changes in the Au film morphology induced by the thermal annealing process. Furthermore, the thermally treated sensor was tested using even lower concentrations of Ara h 1, although label-free detection of Ara h 1 at concentrations as low as $200 \mathrm{nM}$ was previously reported as challenging when using the FO-SPR platform [6], [13]. As can be seen from the obtained calibration curve (Figure 4B), a linear increase of the SPR signal was observed for the entire concentrations domain $(0,25,50,100$ and $200 \mathrm{nM})$. The linear relationship was given by a regression equation with a coefficient of determination $\left(\mathrm{R}^{2}\right)$ of 0.99 . Under these circumstances, the LOD of the thermally annealed sensor was calculated to be $1.6 \mathrm{nM}$ or $0.3 \mu \mathrm{g} / \mathrm{mL}$, with a sensitivity of $0.06 \mathrm{~nm} / \mathrm{nM}$.

The LOD obtained in this work was substantially better compared with the previously reported value (more than $200 \mathrm{nM}$ ) for the non-annealed classical FO aptasensor [6]. In that work, the authors managed to reach a LOD of $75 \mathrm{nM}$ only when using labeling strategies. Furthermore, Peeters et al. [43] reported recently a LOD of $25 \mathrm{nM}$ using a quartz crystal microbalance device, which is still one order of magnitude higher compared with the one presented in this work. Although still 2 times higher (i.e. $3 \mathrm{nM}$ ), a promising result was reported later while employing the heat-transfer technology [44]. However, the same authors showed that the only so-far obtained LOD value of $1 \mathrm{nM}$ was achieved when employing a more complex electrochemical impedance spectroscopy setup [43].

As an overall conclusion, the results achieved in this work clearly demonstrated that the FO-SPR sensor simply exposed to optimal surface thermal treatments may be successfully used for label-free detection of Ara $\mathrm{h} 1$ allergen in the low $\mathrm{nM}$ concentration range. 


\title{
4. Conclusions
}

In this work, the influence of simple thermal treatments on the structural changes of the $\mathrm{Au}$ layer coated on the FO silica core, and consequently on the plasmonic biosensing performance of the as-prepared FO-SPR sensors, was investigated. Carefully conducted refractometric measurements coupled with advanced statistical analysis, suggested an optimal annealing temperature of $180^{\circ} \mathrm{C}$ for a duration of $7 \mathrm{~h}$, where an increase in sensitivity of more than $775 \mathrm{~nm} / \mathrm{RIU}$ was observed. The FO-SPR sensor thermally treated under the optimized parameters was further used as an aptamer - based biosensor for the label-free detection of Ara $\mathrm{h} 1$ peanut allergen. The results demonstrated considerable improvements in the aptasensor's performance, since the resulted LOD was improved with up to two orders of magnitude compared with a similar non-annealed FO-SPR device. Such simple and costeffective thermal treatments are therefore expected to be widely implemented in the preparation of FO-SPR sensing devices, since they could easily result in biosensors with enhanced sensitivity, adequate for label-free detection of various biomolecules.

\section{Acknowledgements}

The authors acknowledge funding from the KU Leuven Industrial Research Fund (IOF, NanoDiag, IOF/KP/12/002) and the Flemish Institute for the Promotion of Innovation through Science and Development (IWT Sensors for Food VIS-trajectory, 100936 and IWTLA project, 110770). K. S. acknowledges support from the Research Foundation - Flanders (FWO, 1270513N, Belgium).

\section{Supporting information}

Supporting information is available and includes: (1) a correspondence table between the sucrose dilutions and the associated refractive index units (RIU), (2) an example of calibration curves measured in serial sucrose dilution, (3) a table summarizing the sensitivity data for all the tested conditions, used further to construct Figure 1, (4) the statistic matched pair t-test outcome, and (5) STM micrographs of the sample annealed at $180^{\circ} \mathrm{C}$ for different durations (2 and $12 \mathrm{~h})$.

\begin{abstract}
Abbreviations
FO-SPR, Fiber Optic - Surface Plasmon Resonance; STM, Scanning tunneling microscope; LOD, Limit of detection; RI(U), Refractive index (units); R RMS, Root mean square roughness.
\end{abstract}




\section{References}

[1] D. R. Shankaran, K. V. Gobi and N. Miura, Recent advancements in surface plasmon resonance immunosensors or detection of small molecules of biomedical, food and environmental interest, Sensors and Actuators B: Chemical 121 (2007) 158-177.

[2] C. Caucheteur, T. Guo and J. Albert, Review of plasmonic fiber optic biochemical sensors: improving the limit of detection, Analytical and bioanalytical chemistry 407 (2015) 3883-3897.

[3] K. Knez, K. P. Janssen, D. Spasic, P. Declerck, L. Vanysacker, C. Denis, D. T. Tran and J. Lammertyn, Spherical Nucleic Acid Enhanced FO-SPR DNA Melting for Detection of Mutations in Legionella pneumophila, Analytical Chemistry 85 (2013) 1734-1742.

[4] S. Rodriguez-Mozaz, M. J. Lopez de Alda and D. Barceló, Biosensors as useful tools for environmental analysis and monitoring, Analytical and bioanalytical chemistry 386 (2006) 1025-1041.

[5] K. Narsaiah, S. N. Jha, R. Bhardwaj, R. Sharma and R. Kumar, Optical biosensors for food quality and safety assurance-a review, Journal of Food Science and Technology 49 (2012) 383-406.

[6] D. T. Tran, K. Knez, K. P. Janssen, J. Pollet, D. Spasic and J. Lammertyn, Selection of aptamers against Ara $\mathrm{h} 1$ protein for FO-SPR biosensing of peanut allergens in food matrices, Biosensors and Bioelectronics 43 (2013) 245-251.

[7] I. Arghir, F. Delport, D. Spasic and J. Lammertyn, Smart design of fiber optic surfaces for improved plasmonic biosensing, New biotechnology 32 (2015) 473-84.

[8] F. Delport, K. Knez, K. Janssen, I. Arghir, N. Marien, D. T. Tran, D. Spasic, S. Vermeir and J. Lammertyn, Aptamer and DNA Hybridization Assays on Gold Fiber Optic Sensors with Nanoparticle Signal Enhancement, Advanced Photonics, OSA Technical Digest (online), Optical Society of America, (2014) JTu1C.2.

[9] M. Zhang, X. -Q. Jiang, H. -N. Le, P. Wang and B. -C. Ye, Dip-and-Read Method for Label-Free Renewable Sensing Enhanced Using Complex DNA Structures, ACS Applied Materials \& Interfaces 5 (2013) 473-478.

[10] Y. S. Dwivedi, A. K. Sharma and B. D. Gupta, Influence of skew rays on the sensitivity and signal-to-noise ratio of a fiber-optic surface-plasmon-resonance sensor: a theoretical study, Applied Optics 46 (2007) 4563-4569.

[11] Q. Zhang, C. Xue, Y. Yuan, J. Lee, D. Sun and J. Xio, Fiber Surface Modification Technology for Fiber-Optic Localized Surface Plasmon Resonance Biosensors, Sensors 12 (2012) 2729-2741.

[12] I. Arghir, D. Spasic, B. E. Verlinden, F. Delport and J. Lammertyn, Improved surface plasmon resonance biosensing using silanized optical fibers, Sensors and Actuators B: Chemical 216 (2015) 518-526. 
[13] J. Pollet, F. Delport, K. P. Janssen, D. T. Tran, J. Wouters, T. Verbiest and J. Lammertyn, Fast and accurate peanut allergen detection with nanobead enhanced optical fiber SPR biosensor, Talanta 83 (2011) 1436-1441.

[14] R. E. P. De Oliveira, N. Sjödin, M. Fokine, W. Margulis, C. J. S. De Matos and L. Norin, Fabrication and Optical Characterization of Silica Optical Fibers Containing Gold Nanoparticles, ACS Applied Materials \& Interfaces 7 (2015) 370-375.

[15] B. D. Gupta and R. K. Verma, Surface Plasmon Resonance-Based Fiber Optic Sensors: Principle, Probe Designs, and Some Applications, Journal of Sensors 2009 (2009) 12 pages.

[16] M. -C. Estevez, M. A. Otte, B. Sepulveda and L. M. Lechuga, Trends and challenges of refractometric nanoplasmonic biosensors: A review, Analytica Chimica Acta 806 (2014) $55-73$.

[17] J. Homola, H. Vaisocherová, J. Dostálek and M. Piliarik, Multi-analyte surface plasmon resonance biosensing, Methods 37 (2005) 26-36.

[18] Y. Hong, Y. -M. Huh, D. S. Yoon and J. Yang, Nanobiosensors Based on Localized Surface Plasmon Resonance for Biomarker Detection, Jounal of Nanomaterials 2012 (2012) 13 pages.

[19] R. Bharadwaj and S. Mukherji, Gold nanoparticle coated U-bend fibre optic probe for localized surface plasmon resonance based detection of explosive vapours, Sensors and Actuators B: Chemical 192 (2014) 804-811.

[20] B. Sciacca and T. M. Monro, Dip Biosensor Based on Localized Surface Plasmon Resonance at the Tip of an Optical Fiber, Langmuir 30 (2014) 946-954.

[21] K. Mitsui, Y. Handa and K. Kajikawa, Optical fiber affinity biosensor based on localized surface plasmon resonance, Applied Physics Letters 85 (2004) 4231.

[22] H. -H. Jeong, N. Erdene, S. -K. Lee, D. -H. Jeong and J. -H. Park, Fabrication of fiberoptic localized surface plasmon resonance sensor and its application to detect antibodyantigen reaction of interferon-gamma, Optical Engineering 50 (2011) 124405-124408.

[23] D. J. Lipomi, R. V. Martinez, M. A. Kats, S. H. Kang, P. Kim, J. Aizenberg, F. Capasso and G. M. Whitesides, Patterning the Tips of Optical Fibers with Metallic Nanostructures Using Nanoskiving, Nano Letters 11 (2010) 632-636.

[24] Y. Lin, Y. Zou, Y. Mo, J. Guo and R. G. Lindquist, E-beam patterned gold nanodot arrays on optical fiber tips for localized surface plasmon resonance biochemical sensing, Sensors (Basel) 10 (2010) 9397-9406.

[25] A. Dhawan, M. D. Gerhold and J. F. Muth , Plasmonic Structures Based on Subwavelength Apertures for Chemical and Biological Sensing Applications, IEEE Sensors Journal 8 (2008) 942-950. 
[26] T. Karakouz, D. Holder, M. Goomanovsky, A. Vaskevich and I. Rubinstein, Morphology and Refractive Index Sensitivity of Gold Island Films, Chemistry of Materials 21 (2009) 5875-5885.

[27] A. Vaskevich and I. Rubinstein, Chapter 14. Localized Surface Plasmon Resonance (LSPR) Transducers Based on Random Evaporated Gold Island Films: Properties and Sensing Applications, in Nanoplasmonic Sensors, A. Dmitriev (Ed.), New York, Springer, 2012, pp. 333-364.

[28] G. Longobucco, G. Fasano, M. Zharnikov, L. Bergamini, S. Corni and M. A. Rampi, High stability and sensitivity of gold nano-islands for localized surface plasmon spectroscopy: Role of solvent viscosity and morphology, Sensors and Actuators B: Chemical 191 (2014) 356-363.

[29] E. Thouti, N. Chander, V. Dutta and V. K. Komarala, Optical properties of Ag nanoparticle layers deposited on silicon substrates, Journal of Optics 15 (2013) 7 pages.

[30] P. Royer, J. P. Goudonnet, R. J. Warmack and T. L. Ferrell, Substrate effects on surfaceplasmon spectra in metal-islands film, Physical Review B 35 (1987) 3753-3759.

[31] U. K. Chettiar, P. Nyga, M. D. Thoreson, A. V. Kildishev, V. P. Drachev and V. M. Shalaev, FDTD modeling of realistic semicontinuous metal films, Applied Physics B 100 (2010) 159-168.

[32] K. I. Mullen and K. T. Carron, Surface-enhanced Raman spectroscopy with abrasively modified fiber optic probes, Analytical Chemistry 63 (1991) 2196-2199.

[33] F. Meriaudeau, A. Wig, A. Passian, T. Downey, M. Buncick and T. L. Ferrell, Gold island fiber optic sensor for refractive index sensing, Sensors and Actuators B: Chemical 69 (2000) 51-57.

[34] Q. Zhong and D. Inniss, Characterization of the lightguiding structure of optical fibers by atomic force microscopy, Journal of Lightwave Technology 12 (1994) 1517-1523.

[35] P. Goos and B. Jones, Optimal Design of Experiments: A Case Study Approach, United Kingdom: John Wiley \& Sons, 2011.

[36] P. Goos, The Optimal Design of Blocked and Split-Plot Experiments, New York: Springer, 2002.

[37] R. Wiesendanger, Scanning Probe Microscopy and Spectroscopy, Cambridge, UK: Cambridge University Press, 1994.

[38] K. Schouteden, E. Lijnen, E. Janssens, A. Ceulemans, L. F. Chibotaru, P. Lievens and C. Van Haesendonck, Confinement of surface state electrons in self-organized Co islands on $\mathrm{Au}(111)$, New Journal of Physics 10 (2008) 043016.

[39] I. Horcas, R. Fernández, J. M. Gómez-Rodríguez, J. Colchero, J. Gómez-Herrero and A. M. Baro, WSXM: A software for scanning probe microscopy and a tool for 
nanotechnology, Review of Scientific Instruments 78 (2007) 013705.

[40] J. B. T. M. Roerdink and A. Meijster, The Watershed Transform: Definitions, Algorithms and Parallelization Strategies, Fundamenta Informaticae 41 (2000) 187-228.

[41] P. Pavaskar, I. -K. Hsu, J. Theiss, W. H. Hung and S. B. Cronin, A microscopic study of strongly plasmonic Au and Ag island thin films, Journal of Applied Physics 113 (2013) 034302 .

[42] L. Nan, D. Allan and L. Gang-yu, In situ STM study of thermal annealing of Au thin films: An investigation on decay of nanometer Au clusters and 2D islands, Acta Physica Sinica (Overseas Edition) 6 (1997) 531.

[43] M. Peeters, K. L. Jiménez-Monroy, C. Libert, Y. Eurlings, W. Cuypers, G. Wackers, S. Duchateau, P. Robaeys, M. Nesládek, B. van Grinsven, E. Pérez-Ruiz, J. Lammertyn, P. Losada-Pérez and P. Wagner, Real-Time Monitoring of Aptamer Functionalization and Detection of Ara H1 by Electrochemical Impedance Spectroscopy and Dissipation-Mode Quartz Crystal Microbalance, Journal of Biosensors \& Bioelectronics 5 (2014) 1000155.

[44] M. Peeters, B. van Grinsven, T. J. Cleij, K. L. Jiménez-Monroy, P. Cornelis, E. PérezRuiz, G. Wackers, R. Thoelen, W. De Ceuninck, J. Lammertyn and P. Wagner, Labelfree Protein Detection Based on the Heat-Transfer Method - A Case Study with the Peanut Allergen Ara h 1 and Aptamer-Based Synthetic Receptors, ACS Applied Materials \& Interfaces 7 (2015) 10316-10323. 


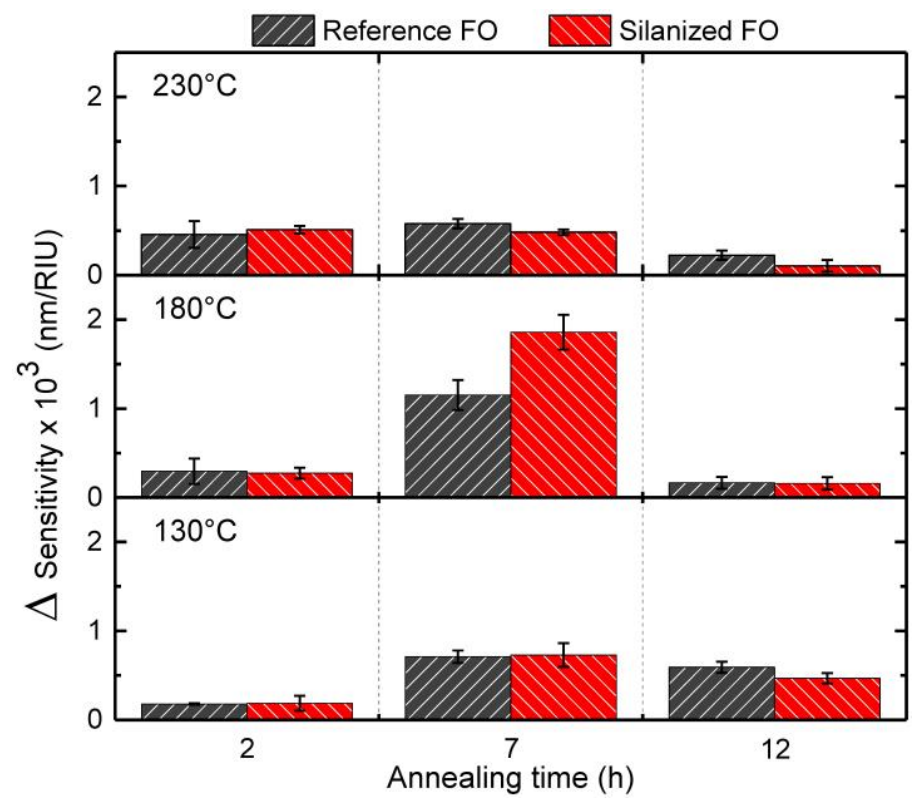

Figure 1. Thermal annealing influence on the FO-SPR sensitivity. Bar charts of the calculated difference in sensitivity $(\Delta)$, before and after the different annealing conditions (130, 180 and $230^{\circ} \mathrm{C}$, for 2,7 and $12 \mathrm{~h}$ ), for reference (black) and silanized (red) FO-SPR sensors. The error bars indicate the standard deviation $(n=3)$.

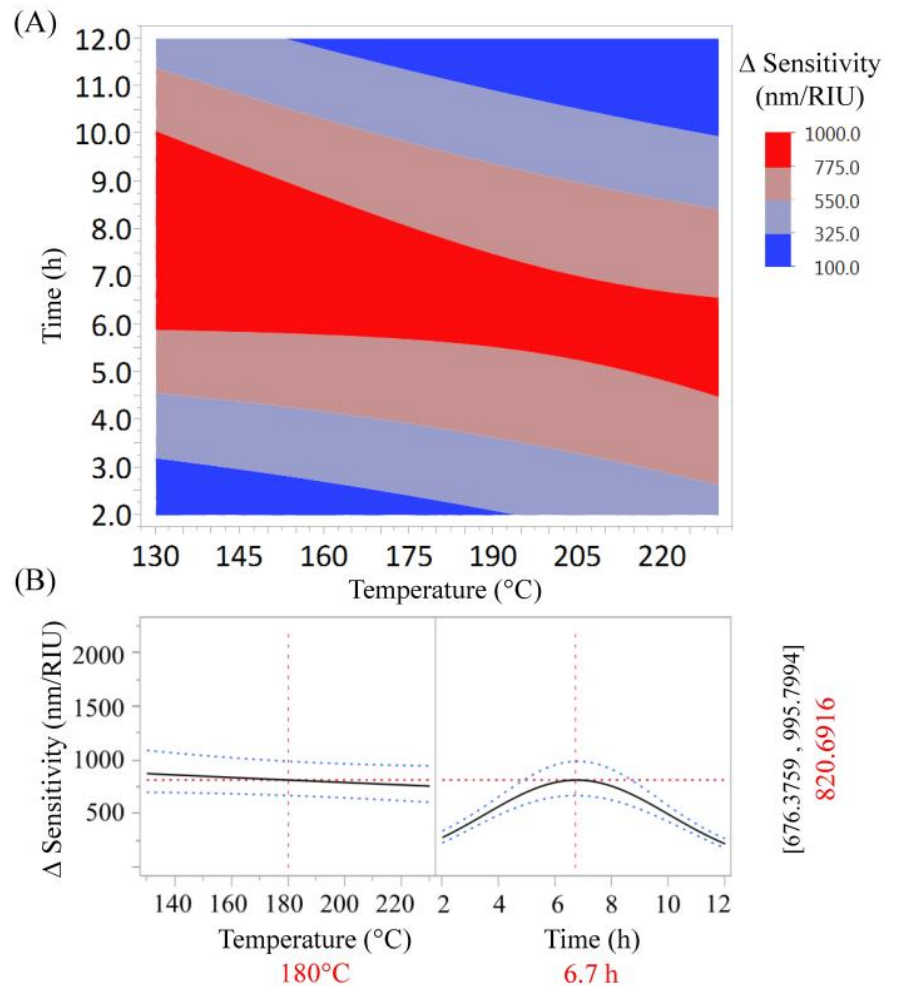

Figure 2. The split-plot experimental design outcome. (A) The response-surface contour plot of the different annealing conditions tested, where the color gradients indicate the predicted $\Delta$ sensitivity values. (B) The prediction profiler estimating the optimal duration at $180^{\circ} \mathrm{C}$. 


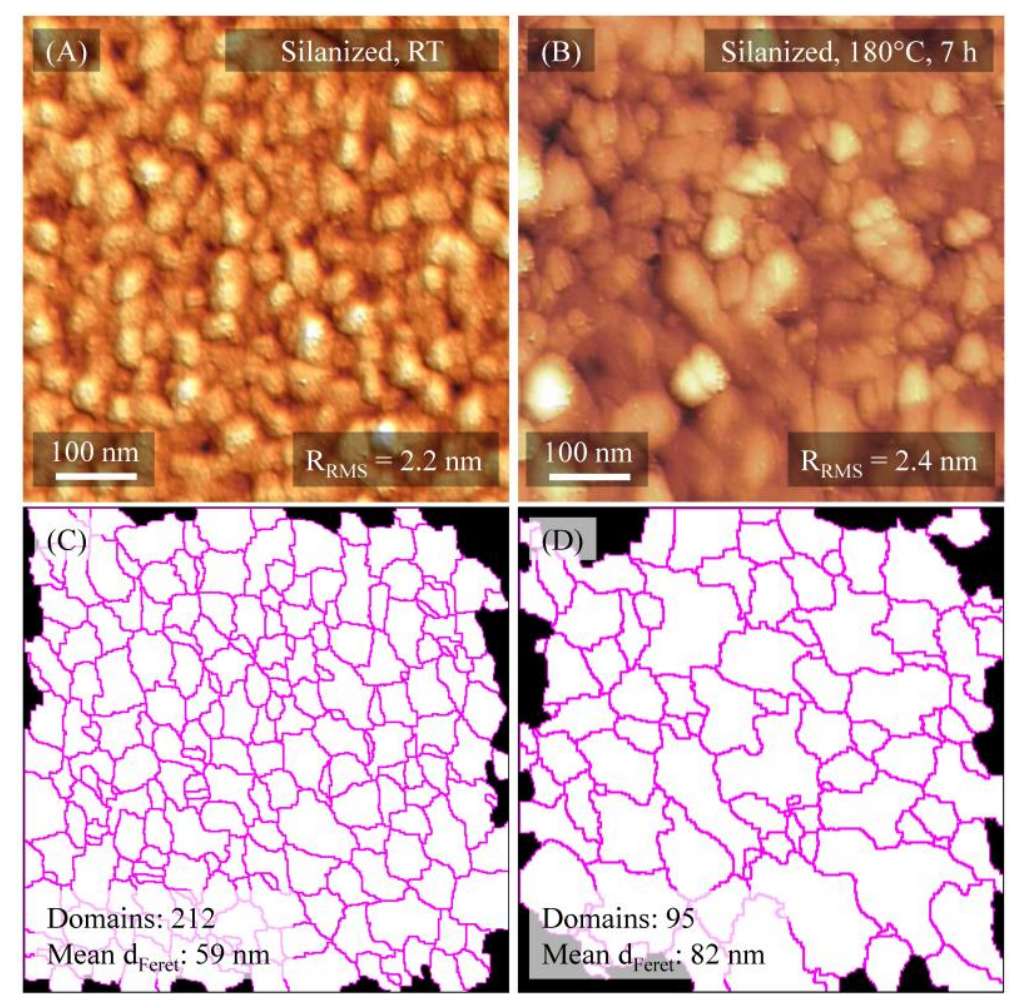

Figure 3. (A, B) STM topographic micrographs $\left(600 \times 600 \mathrm{~nm}^{2}\right)$ of a curved $\mathrm{Au}$ coated silanized FO substrate, before (A) and after (B) exposing it to a thermal annealing process at $180^{\circ} \mathrm{C}$ for $7 \mathrm{~h}$. The images were subject to a planarization algorithm. (C, D) Corresponding segmented images obtained based on a "watershed" algorithm implemented in ImageJ software, showing the Au grains - associated domains before (C) and after (D) the thermal treatment.
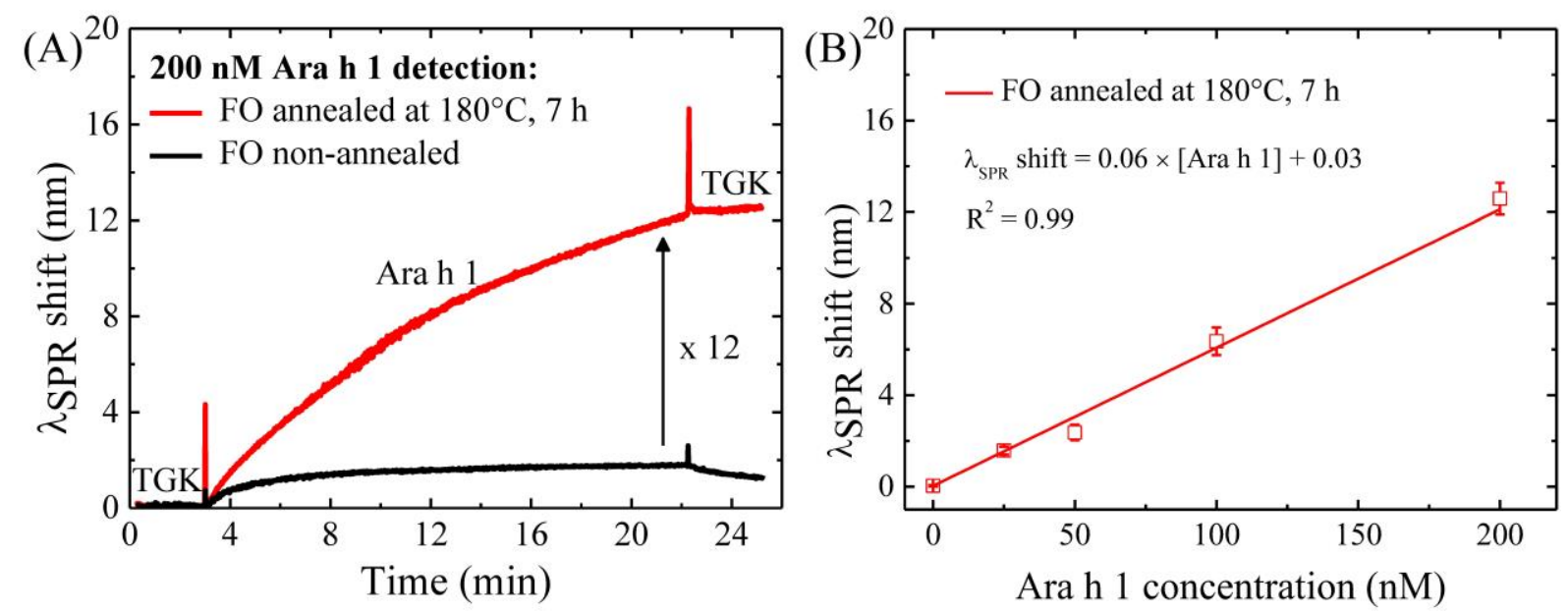

Figure 4. Label-free detection of Ara h 1. (A) SPR sensorgram illustrating the Ara h 1 (200 $\mathrm{nM}$ ) detection using the non-annealed (black) and annealed (red) silanized FO sensors $\left(180^{\circ} \mathrm{C}, 7 \mathrm{~h}\right)$. (B) Linear calibration curve for the thermally annealed FO-SPR aptasensor $\left(\mathrm{R}^{2}\right.$ $=0.99)$. The error bars indicate the standard deviation $(n=3)$. 